\title{
The Numerical Measure of Symmetry for 3D Stick Creatures
}

\author{
Wojciech Jaśkowski and Maciej Komosinski \\ Poznan University of Technology Institute of Computing Science \\ Piotrowo 2, 60-965 Poznań, Poland \\ wjaskowski@cs.put.poznan.pl; Phone: +48 (61) 6652375 \\ maciej.komosinski@cs.put.poznan.pl; Phone: +48 (61) 6652931
}

\begin{abstract}
This work introduces a numerical, continuous measure of symmetry for 3D stick creatures and solid 3D objects. Background information about the property of symmetry is provided, and motivations to developing symmetry measure are described. Three approaches are mentioned, and two of them are presented in detail using a formal mathematical language. The best approach is used to sort a set of creatures according to their symmetry. Experiments with a mixed set of 84 individuals originating from both human design and evolution are performed to examine symmetry within these two sources, and to determine if human designers and evolutionary processes prefer symmetry or asymmetry.
\end{abstract}

Keywords: symmetry, 3D, life form, stick creature, evolution

The final version of this paper appeared in Artificial Life Journal 14(4):425-443, 2008. http://dx.doi.org/10.1162/artl.2008.14.4.14402 


\section{Introduction}

Symmetry is an idea which has guided man through the centuries to the understanding and the creation of order, beauty and perfection-Herman Weyl, "Symmetry".

The ubiquitous symmetry around us is still one of the mysteries of this world. Symmetry is present in physical and mathematical laws and theories, and those considered most beautiful are usually the most symmetrical. Of course, this kind of beauty has a subjective nature.

Mathematically speaking, symmetry is an intrinsic property of a mathematical object which causes it to remain invariant under certain classes of transformations (such as rotation, reflection, inversion, or more abstract operations).

Symmetry has long dominated in architecture and it is an unifying concept for all cultures of the world. Some famous examples include the Pantheon, Gothic churches and the Sydney Opera House.

There is no agreement among scientists why symmetry in biology is such a common evolutionary outcome, but this phenomenon must be certainly related to the properties of the physical world. According to one of the hypotheses [7], a bilaterally symmetrical body facilitates visual perception, as it is easier for the brain to recognize while in different orientations and positions. Another popular hypothesis suggests that symmetry evolved to help with mate selection. It was shown that females of some species prefer males with the most symmetrical sexual ornaments $[8,9]$. For humans, there are proved positive correlations between facial symmetry and health [16], and between facial symmetry and perception of beauty [11].

It is a common intuition that bilateral symmetry resulted from the direction of movement of living creatures. This view was supported by some biological studies suggesting that there is a positive correlation between locomotive efficiency and morphological symmetry $[1,4,12]$. Symmetry of living creatures is only approximate and it is disturbed by such morphological elements as heart that is positioned asymmetrically. It is interesting to note that of all the reports of large scale asymmetry $[10,2]$, none of the investigated asymmetric features directly affected locomotion. On the other hand, in the world of flowers, symmetry (usually radial) is common and it is certainly not related to locomotion.

Symmetry is a very old evolutionary concept. The oldest know bilaterally symmetrical organism is Vernanimalcula that has lived about 600 million years ago. Nowadays, the vast majority of the multicellular organisms exhibit either bilateral or radial symmetry. Radial symmetry is a feature of some marine species like sea anemone, jellyfish, sea stars, etc. Most animals are bilaterally symmetrical (e.g. mammals). Notable exceptions among animals are the sponges.

In this paper, we consider bilateral symmetry for two reasons. First of all, this type of symmetry is the most common in nature and secondly, when a creature is radially symmetrical, it is also bilaterally symmetrical. Therefore, the word "symmetry" used later means a bilateral symmetry, unless stated otherwise. The proposed approach described here can also be employed to evaluating the radial symmetry of creatures and constructs.

\section{The measure of symmetry}

\subsection{Motivations}

There is no objective measure of symmetry. The only thing that can be assessed objectively is whether an object is entirely symmetrical or not. The natural language is not sufficiently precise to express intermediate values of symmetry. We say that something is nearly symmetrical, but we are not able to say that something is symmetrical to a certain degree, and we are not able to specify this degree numerically in the same manner as, for instance, angles can be described. This lack of expressions in natural languages describing partial symmetry is reasonable because, as stated above, many objects in the real world are symmetrical. However, symmetry is not such a common concept in artificial worlds and in order to study the phenomenon of symmetry and its implications, there is a need for defining a numerical, fully automated and objective measure of symmetry for creatures living in artificial environments. 
The natural, "binary" notion of symmetry is insufficient for this application. The advantage of numerical measure of symmetry is not only in that it allows determining the extent to what an object is symmetrical, but also in that it allows to state if one object is more symmetrical than another.

In this paper we address the need to measure symmetry of (artificial) 3D stick creatures or constructs. In the context of artificial life research, this work adds another automatic tool that helps a human examine and evaluate virtual creatures. The numerical measure of symmetry follows the numerical similarity measure of pairs of creatures/constructs [6]. Such measures are especially useful when a researcher faces the need to analyze, systematize or classify large populations or sets of individuals, and these are often encountered in artificial life experiments dealing with evolution and creation. Similarity and symmetry estimates act as simple decision support tools, as they aid a researcher in analysis and interpretation of experimental results. These tools are also helpful for nonprofessionals, as they help reduce complexity and size of experimental data and make it more comprehensible.

\section{$2.2 \quad$ State of work}

A continuous symmetry measure for chemical molecules has been developed in [13, 14]. The same research group defined a symmetry measure for raster images as a quantifier of the minimum 'effort' required to transform a given shape into a symmetric shape [15], but these approaches are not suitable for the model of creatures considered in this report.

The numerical measure of symmetry for artificial creatures was previously considered by Josh Bongard in [3] where the correlation between symmetry and locomotive efficiency for creatures in 3dimensional space was studied. However, that measure of symmetry was defined only for simple treelike creatures consisting of spherical units of identical sizes and masses. Those units could be connected to each other by links of uniform length with no mass. Links between units were constrained to only six cardinal directions. Creatures were facing a fixed direction. In the first step of the algorithm, the plane of symmetry was chosen as a vertical plane that intersects the unit whose horizontal position is closest to the average horizontal positions of all the units. In the second step, the symmetry $\operatorname{sym}(c)$ for creature $c$ was computed using the following equation [3]:

$$
\operatorname{sym}(c)=\frac{4 p l}{(2 n-1)-p-l}
$$

where $n$ was the total number of units creature $c$ consisted of; $2 n-1$ was the total number of units and links creature $c$ consisted of; $p$ was the number of pairs of units lying outside of the plane of symmetry and symmetrical about that plane; and $l$ was the number of pairs of links not contained in the plane of symmetry and symmetrical about that plane.

Let us notice that according to the Eq. (1), if a creature is perfectly symmetrical, then $\operatorname{sym}(c)=1.0$.

The measure described above is sufficient for the simple creature model considered in [3]. Nevertheless, it is not general enough to be successfully applied to more realistic creature models. In this article, a more general measure is proposed.

\subsection{Creature model}

The measure of symmetry introduced in this paper evaluates 3-dimensional creatures consisting of connected, variable length sticks (rods). Creatures can also be equipped with other elements such as receptors, neurons, effectors etc., but only the skeleton (i.e. sticks) is taken into consideration for measure calculation (see Fig. 1). The model resembles stick insects from the order of Phasmatodea, and it can also be used for other creatures with compatible internal structure (e.g. a bone skeleton).

The measure introduced in this paper can also be used for solid 3D objects, like these shown in Fig. 2. In this case, we consider a 3D "mesh" (or a "wireframe") of these objects. Note that the symmetry of a 3D object and its mesh may differ, e.g. a mesh generated for a fully symmetrical sphere by some computer graphics algorithm may be no longer symmetrical.

The measure of symmetry is computed for the creature that is built from its genotype. In an artificial world environment, a creature usually changes its morphology during lifetime - for example, 


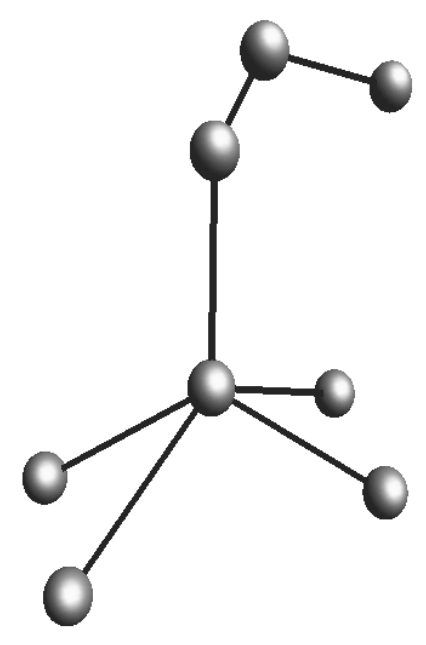

Figure 1: An example of a 3D stick creature. Structures containing cycles (closed loops) are also allowed.

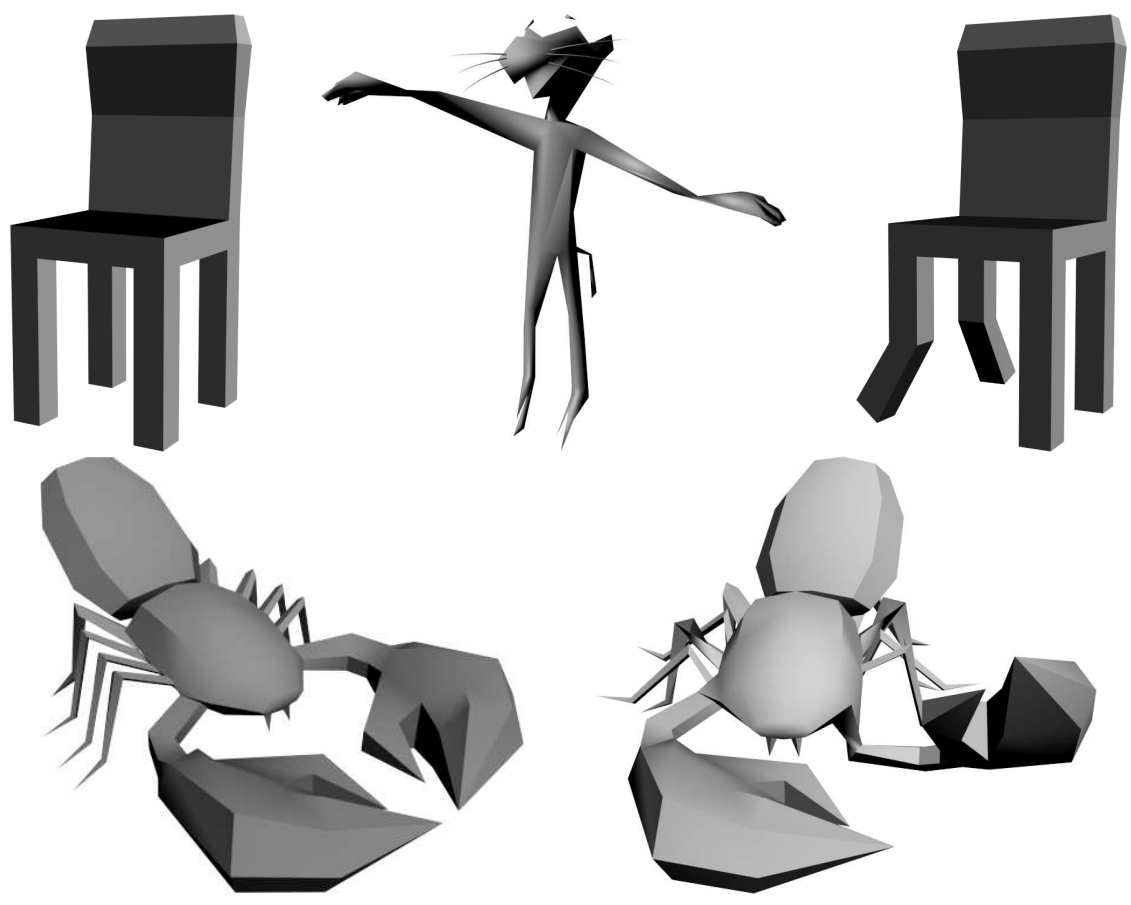

Figure 2: Exemplary solid 3D objects. For their stick (mesh) counterparts, symmetry estimates and symmetry planes, see Fig. 10. Top row: Chair, Pink Panther, Chair crooked. Bottom row: Scorpion, Scorpion moving. 
(a)

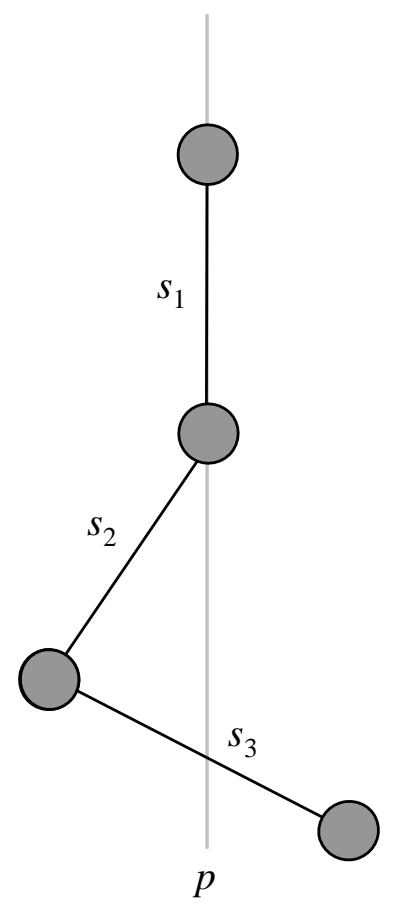

(b)

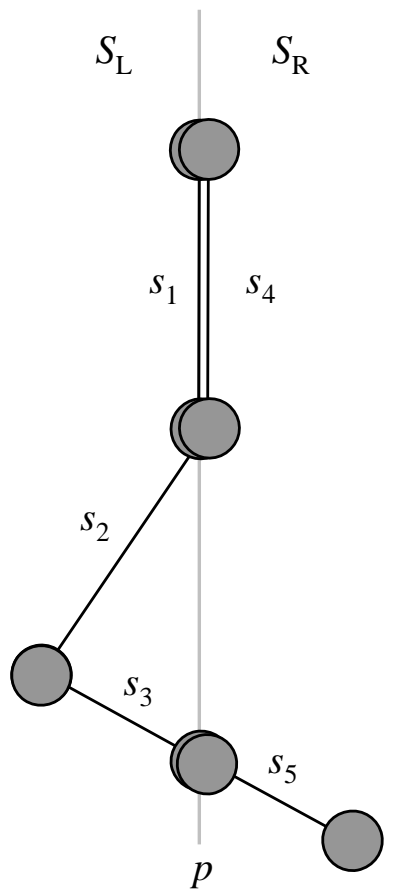

Figure 3: Sticks of a creature (a) are divided into two sets of sticks (b): $S_{L}=\left\{s_{1}, s_{2}, s_{3}\right\}$ and $S_{R}=\left\{s_{4}, s_{5}\right\}$. Note that $s_{4}$ is a copy of $s_{1}$. The naive approach constructs the following pairs: $\Pi=\left\{\left(s_{1}, s_{4}\right),\left(s_{3}, s_{5}\right),\left(s_{2}, s_{\infty}\right)\right\}$.

it uses its muscles to bend some parts of its body. Therefore, it is possible that an initially symmetrical creature would become asymmetrical while interacting with the environment. This situation is also true in the real world, and despite the fact that moving animals are most of the time not symmetrical, we tend to say that they are symmetrical.

\subsection{Notation}

Throughout this article, we use the following naming convention:

$c-$ a creature that is examined for symmetry,

$p \quad-$ a (potential) plane of symmetry,

$S \quad-$ a set of creature's sticks,

$\Pi \quad-\quad$ a set of stick pairs from set $S$,

$s_{i} \quad-$ a stick $\left(s_{i} \in S\right)$

$\operatorname{dist}\left(s_{1}, s_{2}\right)-$ distance between stick $s_{1}$ and stick $s_{2}$ (see Eq. (3)),

$s_{\infty} \quad-$ an "infinity" stick such that $\operatorname{dist}\left(s, s_{\infty}\right)=\infty$ for any $s$, 
$\operatorname{sym}(c) \quad-$ symmetry value of creature $c$ (see Eq. (2)),

$\operatorname{sym}(c, p)$ - symmetry value of creature $c$ about plane $p$ (see Eq. (6) and (5)),

$\operatorname{sim}\left(s_{1}, s_{2}\right)-$ similarity value between stick $s_{1}$ and stick $s_{2}$ (see Eq. (4) and (8)),

$\operatorname{img}(s, p) \quad$ - mirror image of stick $s$ obtained by reflecting $s$ with respect to plane $p$ (see Sec. 3),

$i m g(S, p) \quad$ - set of mirror images of sticks from set $S$ obtained by reflecting each $s \in S$ with respect to plane $p$ (see Sec. 3),

$w_{s_{1} s_{2}} \quad-$ weight of stick pair $\left(s_{1}, s_{2}\right)$ (see Eq. (7)).

This list is for reference only, and each variable and function will be defined where introduced. Note that names sym and sim are used.

\section{Definition of the symmetry measure}

Let us denote the symmetry value of a creature $c$ as $\operatorname{sym}(c)$. We introduce five conditions that $\operatorname{sym}(c)$ is expected to fulfill:

- The Symmetry Condition. If $c$ is perfectly bilaterally symmetrical, then $\operatorname{sym}(c)=1.0$.

- The Asymmetry Condition. If $c$ is completely asymmetrical ${ }^{1}$, then $\operatorname{sym}(c)=0.0$.

- The Common Sense Condition. If $c_{1}$ is more symmetrical than $c_{2}$, then $\operatorname{sym}\left(c_{1}\right)>\operatorname{sym}\left(c_{2}\right)$.

- The Proportional Difference Condition. The difference between $\operatorname{sym}\left(c_{1}\right)$ and $\operatorname{sym}\left(c_{2}\right)$ should correspond to the difference in anatomical symmetry between $c_{1}$ and $c_{2}$.

- The Scalability Condition. The proposed measure should be robust against scaling: for creature $c_{2}$ that is a scaled version of $c_{1}$ (body enlarged or diminished), we expect $\operatorname{sym}\left(c_{2}\right)=\operatorname{sym}\left(c_{1}\right)$.

It is important to note that although The Symmetry Condition and The Scalability Condition can be verified in an objective way, The Common Sense Condition and The Proportional Difference Condition are of a subjective, perceptive nature.

Let us denote symmetry of a creature $c$ about plane $p$ as $\operatorname{sym}(c, p)$. We say that "a creature is symmetrical" if it is symmetrical about any plane, therefore we are looking for a plane that yields the highest symmetry:

$$
\operatorname{sym}(c)=\max _{p}(\operatorname{sym}(c, p))
$$

Two approaches will be shown to defining $\operatorname{sym}(c, p)$. We will present an intuitive (naive) approach, discuss its disadvantages, describe improvements and then show the final approach.

One approach that is not described in this report was based on statistical distribution of parts of a creature body in the 3D space. This approach was based on the principal assumption that the plane of symmetry is a regression plane that minimizes the distance from all creature parts. Although computationally efficient and usually successful, this approach was abandoned as the assumption was not true in general.

\footnotetext{
${ }^{1}$ It is hard to imagine the "completely asymmetrical" creature, so the symmetry value of zero may be unreachable. As an example, let us consider another setting: a (multidimensional) grid with black and white cells. The lowest bilateral symmetry configuration would be the uniform random noise. In this configuration, the location of the symmetry (hyper)plane would be irrelevant, and the lowest expected value of symmetry (computed as the fraction of corresponding cells with differing colors) would be 0.5 .
} 


\subsection{Naive approach}

\subsubsection{Two sides of the plane - step 1}

Having a plane $p$, sticks are divided into two groups: $S_{L}$ - sticks that lie on the left side of $p$ and $S_{R}$ - sticks that lie on the right side of $p$. There are, however, two special cases. The first case is when a stick crosses $p$. In this situation, the stick is divided into two parts that are treated as two different sticks belonging to the appropriate sets $\left(S_{L}\right.$ and $\left.S_{R}\right)$. The second special case is when a stick is completely contained in $p$. In this situation, a copy of the stick is put into the first set (e.g. $\left.S_{R}\right)$ whereas the original one is put into the second set (e.g. $\left.S_{L}\right)$. See Fig. 3.

\subsubsection{Evaluating symmetry - step 2}

In is intuitive that the more correspondence there is between sticks from $S_{L}$ and sticks from $S_{R}$, the more symmetrical the creature is. The best correspondence is the one that yields highest symmetry, and this correspondence needs to be found. Let the distance between two sticks be given by:

$$
\operatorname{dist}\left(s_{1}, s_{2}\right)=\min \left(d\left(s_{1}^{a}, s_{2}^{a}\right)+d\left(s_{1}^{b}, s_{2}^{b}\right), d\left(s_{1}^{a}, s_{2}^{b}\right)+d\left(s_{1}^{b}, s_{2}^{a}\right)\right)
$$

where $s^{a}$ and $s^{b}$ are the two vertices of the stick $s$, whereas $d(k, l)$ is the Euclidean distance between points $k$ and $l$.

For the sake of simplicity of the following definitions, we assume that there exists a special "infinity" stick $s_{\infty}$ such that for any $s, \operatorname{dist}\left(s, s_{\infty}\right)=\infty$.

Similarity between two sticks is then given by:

$$
\operatorname{sim}\left(s_{1}, s_{2}\right)=\frac{1}{1+\beta \cdot \operatorname{dist}\left(s_{1}, s_{2}\right)}
$$

When the distance between sticks is 0 , the similarity of these sticks is 1 . On the other hand, when the distance between sticks approaches infinity, their similarity converges to 0 . $\beta$ was introduced to control the sensitivity of sim to dist, and based on experiments it was adjusted to 10 .

Let $i m g(s, p)$ be a mirror image of stick $s$ obtained by reflecting $s$ with respect to plane $p$. Similarly, let $i m g(S, p)$ be a set of mirror images of sticks from set $S$ reflected with respect to plane $p$.

Now, similarity $\operatorname{sim}\left(s_{1}, s_{2}\right)$ is computed for $s_{1} \in S_{L}$ and $s_{2} \in i m g\left(S_{R}, p\right)$. Intuitively, the more sticks from set $S_{L}$ are similar to sticks from set $i m g\left(S_{R}, p\right)$, the more symmetrical the whole creature is about plane $p$. Thus, from all possibilities of creating stick pairs, the best one is the one that maximizes the total symmetry:

$$
\operatorname{sym}(c, p)=\max _{\Pi}\left(\frac{\sum_{\left(s_{1}, s_{2}\right) \in \Pi} \operatorname{sim}\left(s_{1}, s_{2}\right)}{|\Pi|}\right)
$$

where $\Pi$ is a set of pairs $\left(s_{1}, s_{2}\right)$ of sticks with $s_{1} \in S_{L} \cup\left\{s_{\infty}\right\}$ and $s_{2} \in i m g\left(S_{R}, p\right) \cup\left\{s_{\infty}\right\}$. It is important that each element of $S_{L}$ occurs exactly once as the first element of pair in $\Pi$ and each element of $S_{R}$ occurs exactly once as the second element of pair in $\Pi$. The special stick $s_{\infty}$ can occur multiple times as both elements of pairs, with the exception of the $\left(s_{\infty}, s_{\infty}\right)$ pair which is not allowed.

\subsubsection{Discussion}

It is clear that if creature $c$ is fully symmetrical, then, according to the above definitions, $\operatorname{sym}(c)=1.0$. If there is no good correspondence for any plane between sticks from set $S_{L}$ and set $S_{R}$ (i.e. there is little similarity between sticks from sets $S_{L}$ and $\operatorname{img}\left(S_{R}, p\right)$ ), then $\operatorname{sym}(c)$ will be less than 1.0.

However, this approach has a serious disadvantage. Let us suppose that creature $c$ has a stick $s$ which is completely contained in the potential plane of symmetry $p_{1}$. Then, $\Pi$ will contain a pair $(s, \operatorname{copy}(s))$, where $s=\operatorname{copy}(s)$ (but $s \in S_{L}$ and $\left.\operatorname{copy}(s) \in S_{R}\right)$. Of course, $\operatorname{sim}\left(s, \operatorname{img}\left(\operatorname{copy}(s), p_{1}\right)\right)=$ 1.0. The contribution of this pair to the value of $\operatorname{sym}\left(c, p_{1}\right)$ is therefore maximum. 
(a)

(b)
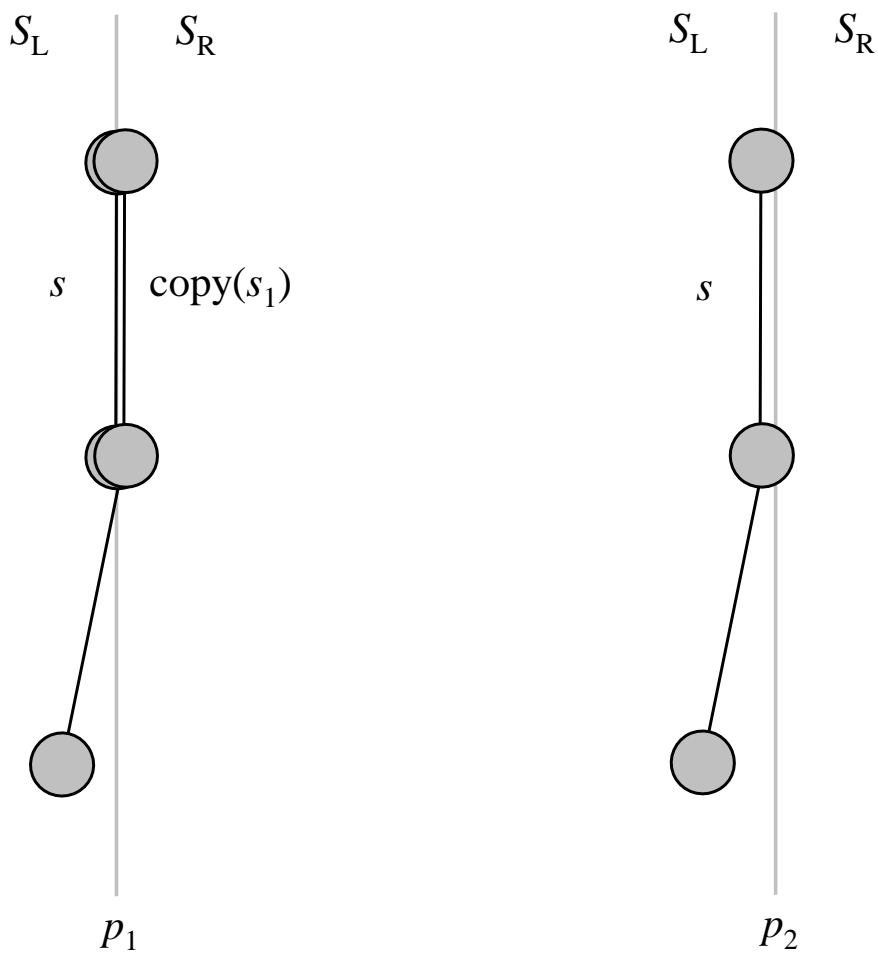

Figure 4: Disadvangate of the naive approach. (a) Stick $s$ is contained in plane $p_{1}$, thus it is paired with its copy. This pair is very likely to increase the value of $\operatorname{sym}\left(c, p_{1}\right)$. (b) If the plane is moved a little bit, then the stick can only be paired with $s_{\infty}$. 
(a)

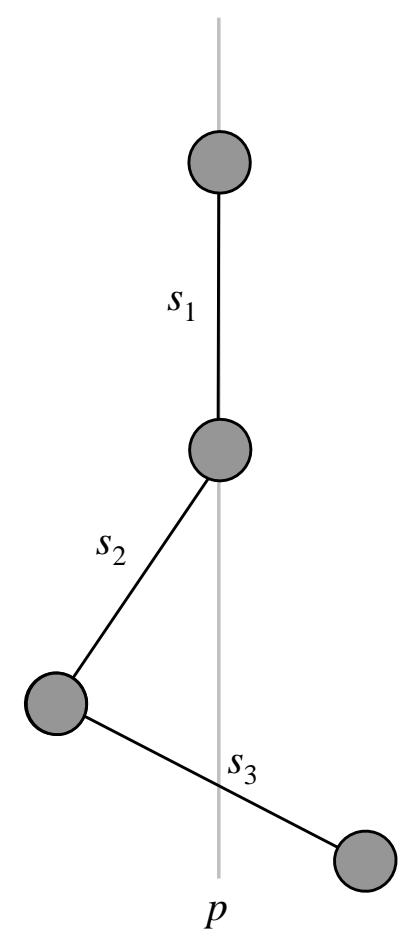

(b)

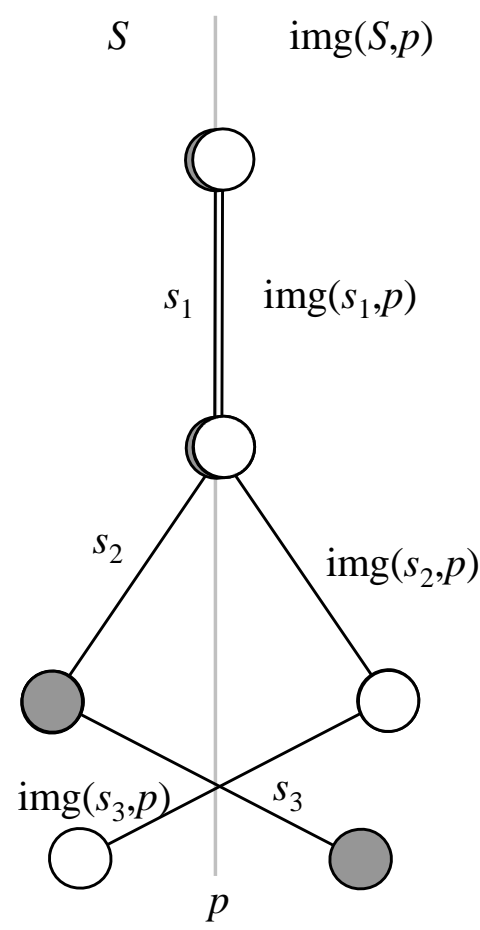

Figure 5: Fig. (a) shows the original set of sticks $S$. Fig. (b) additionally shows image sticks $\operatorname{img}(S, p)$. The final approach constructs the following pairs: $\Pi=$ $\left\{\left(s_{1}, i m g\left(s_{1}, p\right)\right),\left(s_{2}, i m g\left(s_{3}, p\right)\right),\left(s_{3}, i m g\left(s_{2}, p\right)\right\}\right.$.

Now let us suppose that we have another plane $p_{2}$ near $p_{1} . s$ is not contained in $p_{2}$, but it is very close to $p_{2}$. In this case, $s$ is a member of one of the sets (e.g. $S_{L}$ ), and $\Pi$ would contain a pair $\left(s, s_{x}\right)$, where $s_{x} \in \operatorname{img}\left(S_{R}, p_{2}\right) \cup\left\{s_{\infty}\right\}$. Now $\operatorname{sim}\left(s, s_{x}\right)$ may be much lower then 1.0 (it would be 0 for one-stick creature, because then $\left.s_{x}=s_{\infty}\right)$. In consequence, $\operatorname{sim}\left(s, s_{x}\right)$ is likely to decrease the value of $\operatorname{sym}\left(c, p_{2}\right)$. As we see, although the distance between planes $p_{1}$ and $p_{2}$ is very small, the difference between $\operatorname{sym}\left(c, p_{1}\right)$ and $\operatorname{sym}\left(c, p_{2}\right)$ can be significant (the difference would be 1.0 for one-stick creature). See Fig. 4 for illustration.

The above observations have severe implications. Firstly, even small differences in creature anatomy can significantly change $\operatorname{sym}(c)$. Secondly, such a discontinuity of $\operatorname{sym}(c, p)$ makes implementation of an algorithm that finds best plane $p$ (according to Eq. (2)) difficult.

\subsection{The final approach}

In this section we introduce an improved similarity definition addressing problems mentioned in the previous section.

The final approach is similar to the naive one in that it also tries to create pairs of corresponding sticks in the optimal way. However, the construction method is different. This time the set of sticks $S$ is not divided into two sets $S_{L}$ and $S_{R}$. The most important difference between the two approaches is that each stick from set $S$ can be assigned to any of the sticks from the set $i m g(S, p)$ - see Fig. 5 . 
(a)

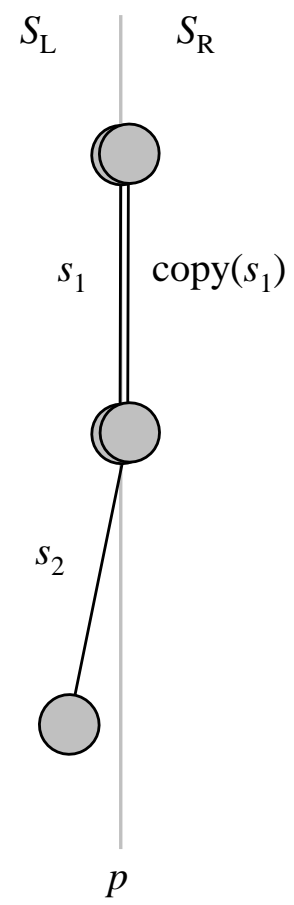

(b)

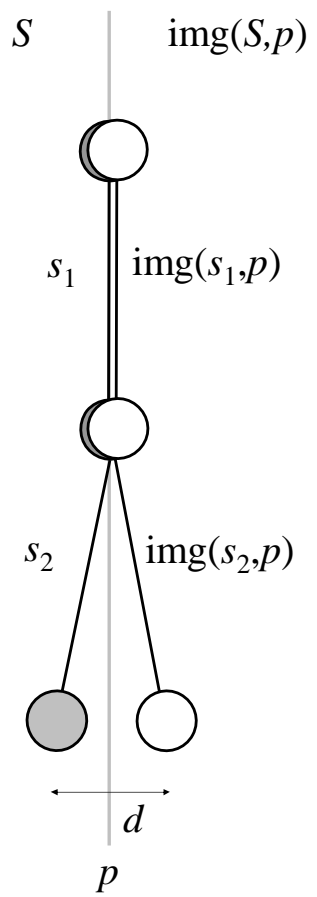

Figure 6: The difference between the naive approach (a) and the final one (b). In the naive approach $\operatorname{sym}(c, p)=0.5$, because $s_{1}$ is paired with $\operatorname{copy}\left(s_{1}\right)$ (with similarity of 1.0) and $s_{2}$ is paired with $s_{\infty}$ (with similarity of 0 ). In the final approach, $s_{1}$ is paired with $i m g\left(s_{1}, p\right)$ (with similarity of 1.0) and $s_{2}$ is paired with $i m g\left(s_{2}, p\right)$ with similarity 0.5 (for $d=0.1$ ), thus $\operatorname{sym}(c, p)=0.75$, which is closer to the expected value of symmetry for this highly symmetrical creature.

Thus, the definition of the set $\Pi$ in Eq. (4) changes to:

$\Pi$ is a set of pairs $\left(s_{1}, s_{2}\right)$ of sticks where $s_{1} \in S$ and $s_{2} \in i m g(S, p)$. Each element of $S$ occurs in exactly one pair in $\Pi$ (more precisely, $\forall_{s \in S} \exists !_{\left(s_{1}, s_{2}\right) \in \Pi}: s_{1}=s \vee s_{2} \in i m g(s, p)$ ). A stick $s$ can be assigned to its own image $(s, i m g(s, p))$ which makes sense for sticks contained in the plane $(\operatorname{sim}(s, i m g(s, p))=$ 1.0), but also for sticks that are very close to the plane and there is no corresponding stick on the other side (then $\operatorname{sim}(s, i m g(s, p))<1.0$, but it will still be high).

This final approach has two advantages. It preserves continuity when moving the plane as it was shown in Sec. 3.1.3, which is generally desirable. It also makes it easier to find the solution of Eq. 5 (see Sec. 4.2 for details). Fig. 6 illustrates the advantage of the final approach over the naive one.

\subsubsection{Stick weights}

So far, all sticks were treated equally (they equally influenced the total symmetry, as in Eq. 5). This would be sufficient if all sticks had the same length, but the creature model we consider is more general and it allows for sticks of various lengths. Therefore, we change the sum from Eq. (5) into a weighted sum to take into consideration stick lengths: 

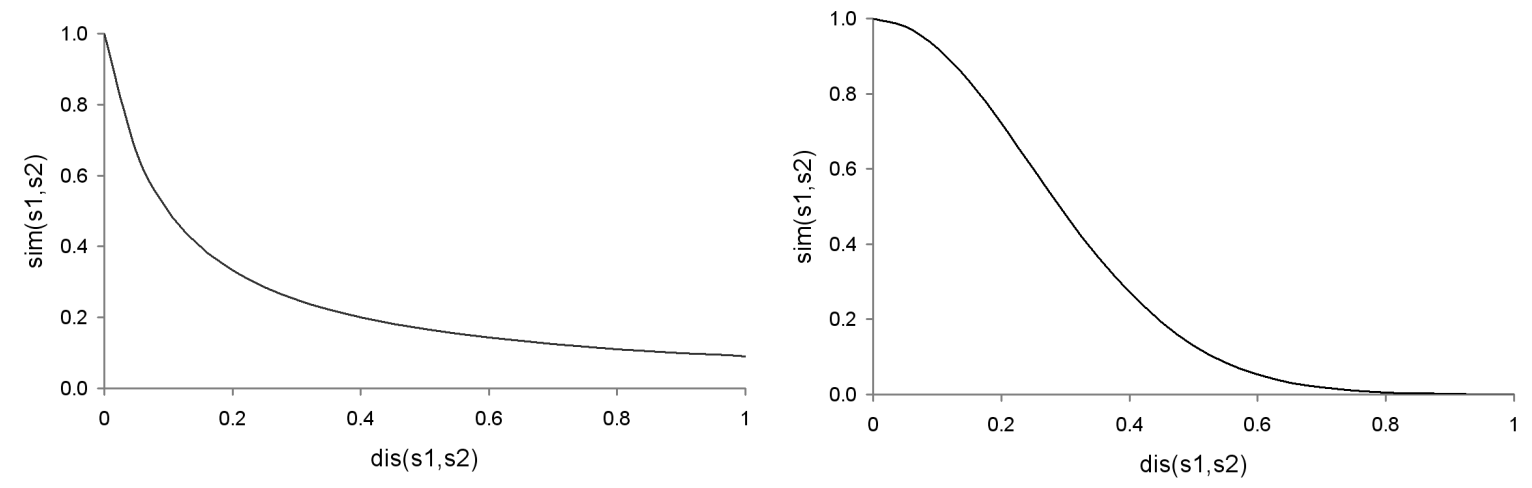

Figure 7: Two characteristics of $\operatorname{sim}\left(s_{1}, s_{2}\right)$. The typical stick length is 1.0. The function on the left illustrates Eq. (4) for $\beta=10$. The right plot presents the improved version of similarity definition, as in Eq. (8) for $\alpha=0.17$ and $s_{f}=2.06$.

$$
\operatorname{sym}(c, p)=\max _{\Pi}\left(\frac{\sum_{\left(s_{1}, s_{2}\right) \in \Pi} w_{s_{1} s_{2}} \operatorname{sim}\left(s_{1}, s_{2}\right)}{\sum_{\left(s_{1}, s_{2}\right) \in \Pi} w_{s_{1} s_{2}}}\right)
$$

where

$$
w_{s_{1} s_{2}}=\left\{\begin{array}{ccc}
\operatorname{len}\left(s_{1}\right)+\operatorname{len}\left(s_{2}\right) & \text { if } & s_{1} \neq s_{2} \\
\operatorname{len}\left(s_{1}\right) & \text { if } & s_{1}=s_{2}
\end{array}\right.
$$

It is possible to define $w_{s_{1} s_{2}}$ in a different way to take into account other properties of sticks (e.g. mass) that are important in a particular creature model.

\subsubsection{Stick similarity and creature size}

In paragraph 3.1.2 we assumed that stick similarity can be defined by Eq. (4). The characteristic of this function is shown in Fig. 7, left. This function has two drawbacks: firstly, it changes too fast when the distance between sticks increases from 0 to 0.2 and it is nearly constant for distances bigger than 0.4 , and secondly, it is hard to take scaling (creature size) into account. Therefore, we introduce a Gauss-like similarity function (see Fig. 7) defined as follows:

$$
\operatorname{sim}\left(s_{1}, s_{2}\right)=\exp \frac{-d i s t^{2}\left(s_{1}, s_{2}\right)}{\left(\alpha \cdot s_{f}\right)^{2}}
$$

where $\alpha$ is a constant, and $s_{f}$ is a creature scale factor.

The purpose of $s_{f}$ (and $\alpha$ ) is to make the similarity measure robust against changes in creature size. Generally, the scale factor should take into account the number of sticks and stick lengths of a creature. We assumed that $s_{f}=\left(\sum_{s \in S} \operatorname{len}(s)\right)^{1 / 3}$, as this formula ensures that values of similarity do not vary while the scale of the creature changes. Based on experiments, the value of $\alpha$ was adjusted to 0.17 .

\section{Algorithms}

As all the necessary definitions have been introduced, this section will show how to effectively implement the final approach - Equations $(2,6)$. The implementation is not straightforward because of two problems: 
1. In order to determine the best set of stick pairs $\Pi$ in Eq. (6), all possible sets of stick pairs should be examined. That would mean the time complexity of $O(n !)$ where $n=|S|$. We use a greedy heuristic instead.

2. It is not possible to find the best plane $p$ in Eq. (2) analytically. We use a heuristic algorithm for this purpose.

\subsection{Determining the best set of stick pairs $\Pi$}

To determine the best set of stick pairs $\Pi$ according to the Eq. (6), we start with an empty set and add iteratively a new pair of sticks $\left(s_{1}, s_{2}\right)$ that maximizes $w_{s_{1} s_{2}} \operatorname{sim}\left(s_{1}, s_{2}\right)$. This is a greedy approach and it does not guarantee the best possible set of stick pairs $\Pi$, but, as experiments have shown, its quality is sufficient in practice. The implementation uses a sorted list for best performance, so the overall time complexity of this procedure is $O\left(n^{2} \log n\right)$ where $n=|S|$.

\subsection{Finding the best plane of symmetry}

According to Eq. (2), we have to find a plane that maximizes symmetry of the creature. The general equation of a plane is $a x+b y+c z+d=0$. Using this representation of a plane would require searching the 4 -dimensional space of parameters $(a, b, c, d)$. This approach has three major disadvantages. First, there is some redundancy and dependence among the four parameters, because a plane can be defined by only three parameters. Second, the parameters are not limited: $a, b, c, d \in[-\infty,+\infty]$. Third, the influence of each parameter on the orientation of the plane varies depending on the magnitude of the parameter. This makes the problem more difficult for optimization algorithms.

However, each plane $p$ can also be defined by a vector $v$ perpendicular to $p$ and a point $t$ contained by $p$. $v$ is represented by two angles ${ }^{2}$ : azimuth $\alpha$ and altitude $\beta$. As the direction of $v$ is not important, both $\alpha$ and $\beta$ can be constrained to $[0, \pi)$. The location of $t$ can be limited to the points that belong to creature sticks. Thus for some stick $s, t$ is unambiguously determined by a relative position $r \in[0,1]$ within $s$. For $r=0$, the point $t$ is in the first endpoint of the stick $s$. For $r=0.5, t$ is the middle of $s$, etc.

The latter representation of a plane is much better for our purposes: it is non-redundant, the range of parameters is limited, and the influence of each parameter on the orientation of the plane does not depend on its magnitude. It also yields smooth landscapes of the optimized function $\operatorname{sym}(c, p)$, as shown in Fig. 8, which facilitates the process of finding the best plane of symmetry.

In order to find the plane of the highest symmetry, we sample the 3 -dimensional $(\alpha, \beta, t)$ space for each creature stick and then perform a local search to further improve the best found plane.

\section{$5 \quad$ Experiments}

Numerous experiments were performed in order to test the numerical measure of symmetry. We used phenotypes created (designed, evolved) in the Framsticks environment [5], as there is a large database of diversified creatures available. This database includes both results of various evolutionary experiments and the most interesting constructs designed by hand, as well as designed individuals that underwent evolution.

\subsection{Illustration of planes of symmetry}

Fig. 9 shows planes of symmetry computed using the final approach described previously. The planes shown are those that yield the maximum overall value of symmetry. Seven test creatures were selected ranging from simple constructs to more sophisticated ones. The top row displays clearly symmetrical individuals (the top right construct with the symmetry of 0.99 is not perfectly symmetrical). In the

\footnotetext{
${ }^{2}$ As in the horizontal coordinate system.
} 

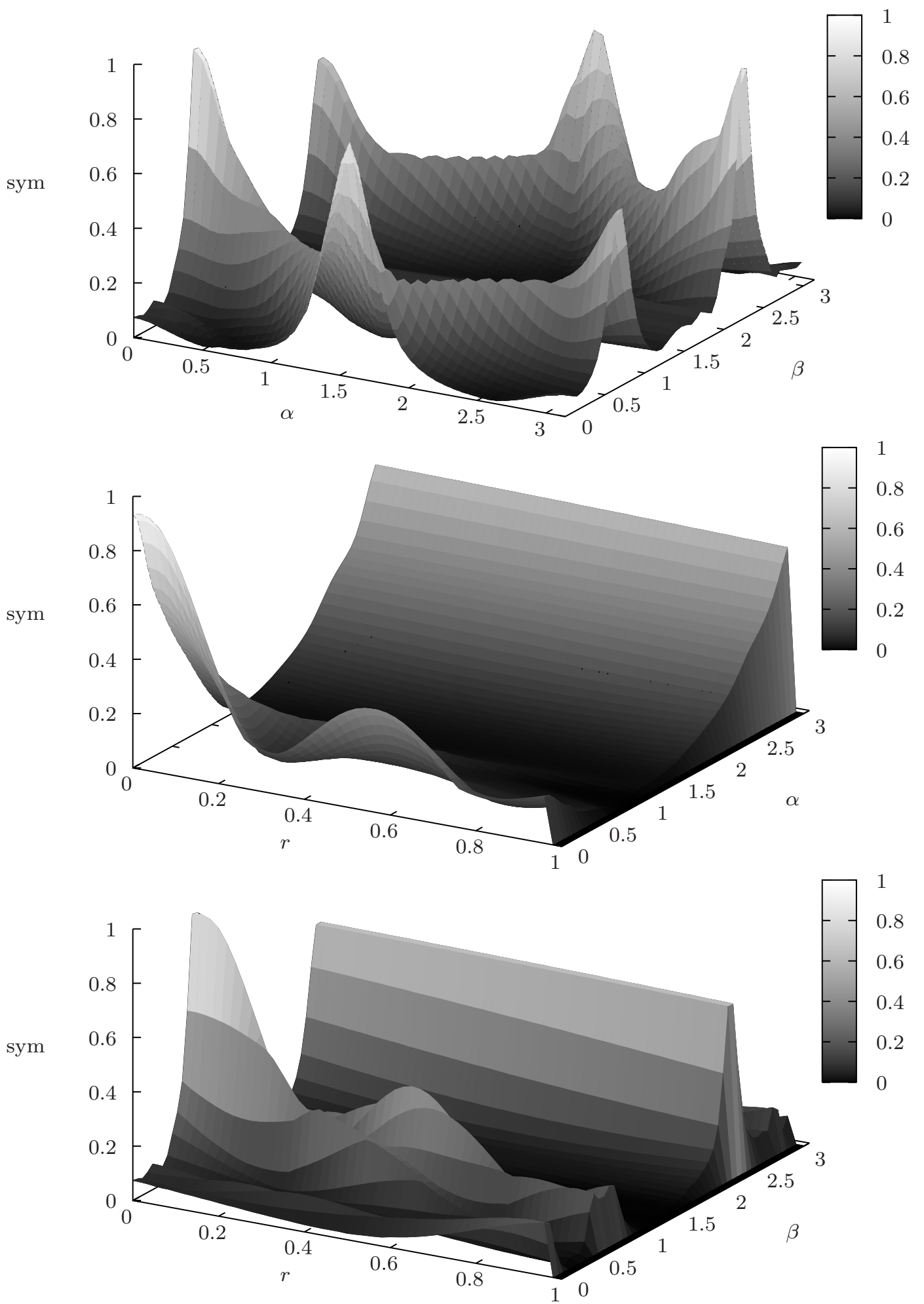

Figure 8: Sample landscapes of symmetry value $\operatorname{sym}(c, p)$ for two selected parameters. Plots show intersections of the 3 -dimensional $(\alpha, \beta, r)$ parameter space. 

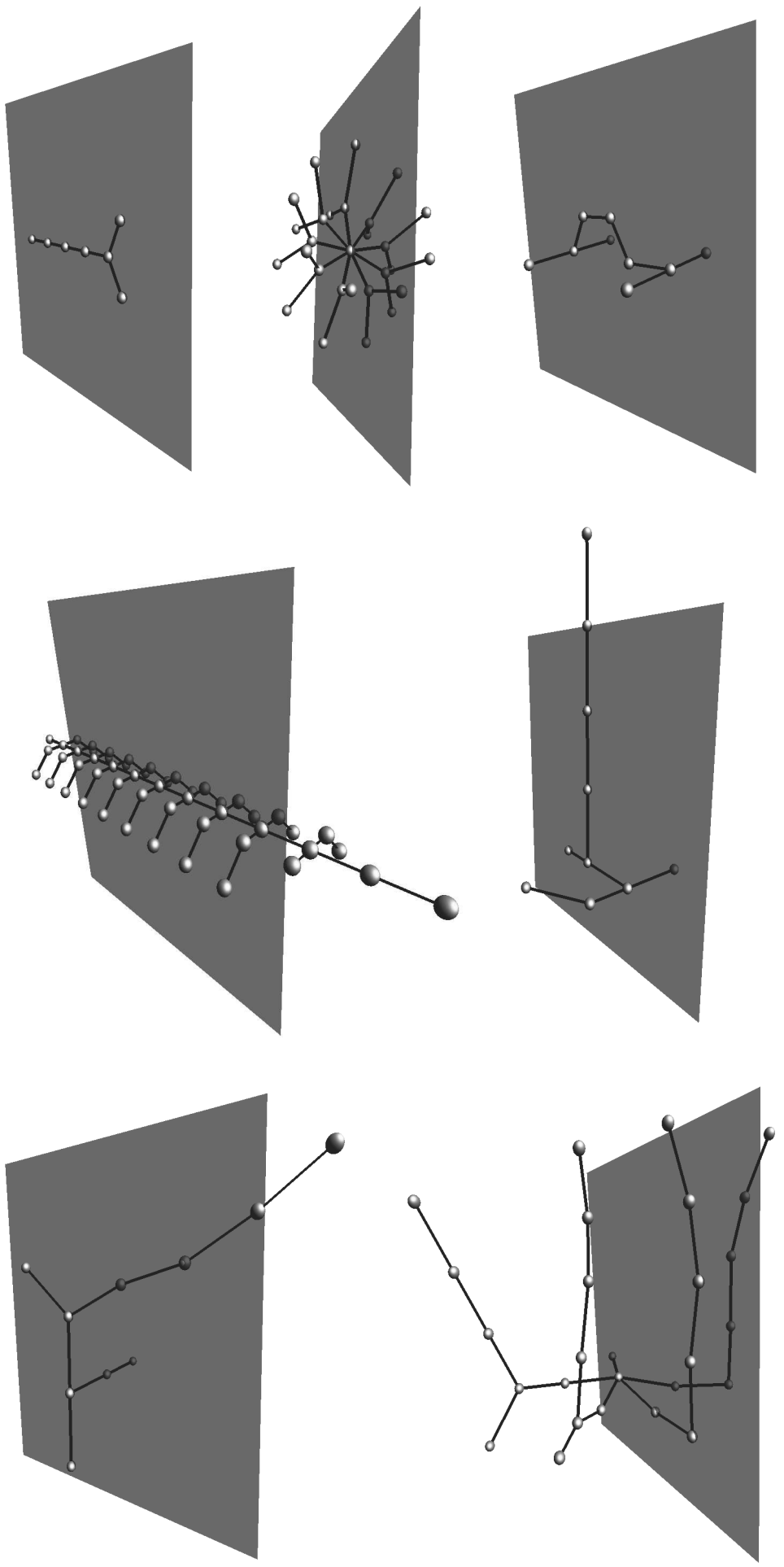

Figure 9: Exemplary creatures, estimation of their symmetry planes and symmetry values. Values of symmetry are: (top) 1.0, 1.0, 0.99; (middle) 0.97, 0.82; (bottom) 0.70, 0.39 


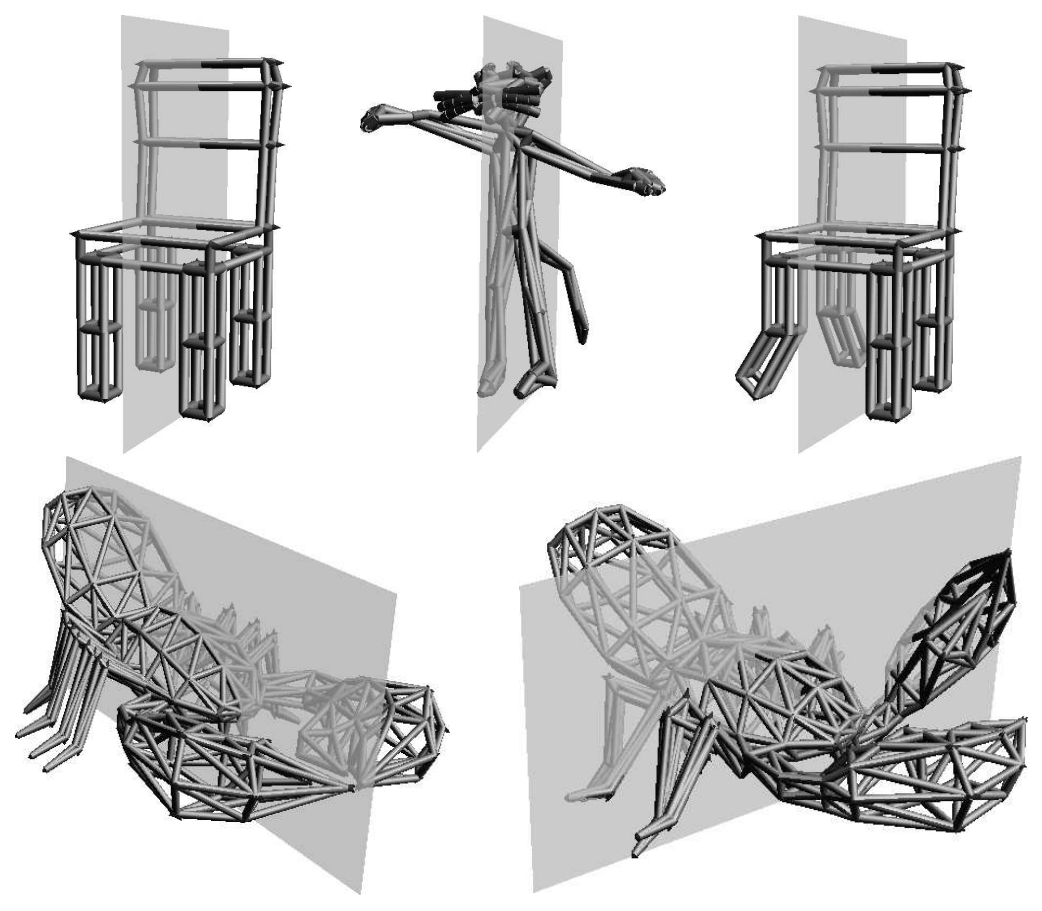

Figure 10: Stick counterparts of solid objects shown on Fig. 2 and highest symmetry planes. Symmetry values are as follows. Top row: Chair (1.0), Pink Panther (0.84), Chair crooked (0.92). Bottom row: Scorpion (1.0), Scorpion moving (0.82).

middle row, the left "Centipede" creature has one leg shorter (missing segment) which results in the symmetry of 0.97 . The middle right structure has an asymmetrical base which yields the symmetry of 0.82 . Two individuals in the bottom row are generally asymmetrical, but the algorithm finds the plane that gives the highest symmetry, and it is expressed in values 0.70 and 0.39 , respectively. Results presented in Fig. 9 are consistent with the common sense.

Fig. 10 shows planes of symmetry computed for meshes of solid objects from Fig. 2. Note how the specific movement of body parts of "Scorpion" disturbed the highest symmetry plane, which became perpendicular for "Scorpion moving".

\subsection{A random set of individuals}

To test the symmetry measure on a larger set of creatures, we selected 30 diverse phenotypes from those available in the database. This testing set contains diversified constructs: small, big, symmetric, asymmetric, human-designed and evolved. These creatures are arranged according to computed values of their symmetry in Fig. 11, where the horizontal axis shows values of symmetry. The creatures are oriented such that the plane of symmetry for each of them is a vertical plane perpendicular to the horizontal axis. Constructs that were designed (not evolved) and have regular shapes are the most symmetrical ones (located on the right side with symmetry close to 1.0). On the other hand, large evolved bush-like creatures, for which symmetry planes were not obvious, are located on the far left (low values of symmetry).

\subsection{Symmetry in human design and in evolution}

Once we are able to measure symmetry, a question comes up about the symmetry of human designs compared to the symmetry of evolved constructs. To investigate this issue, a set of 84 representative creatures has been selected from the genotype database. 38 of them were designed by a human, 


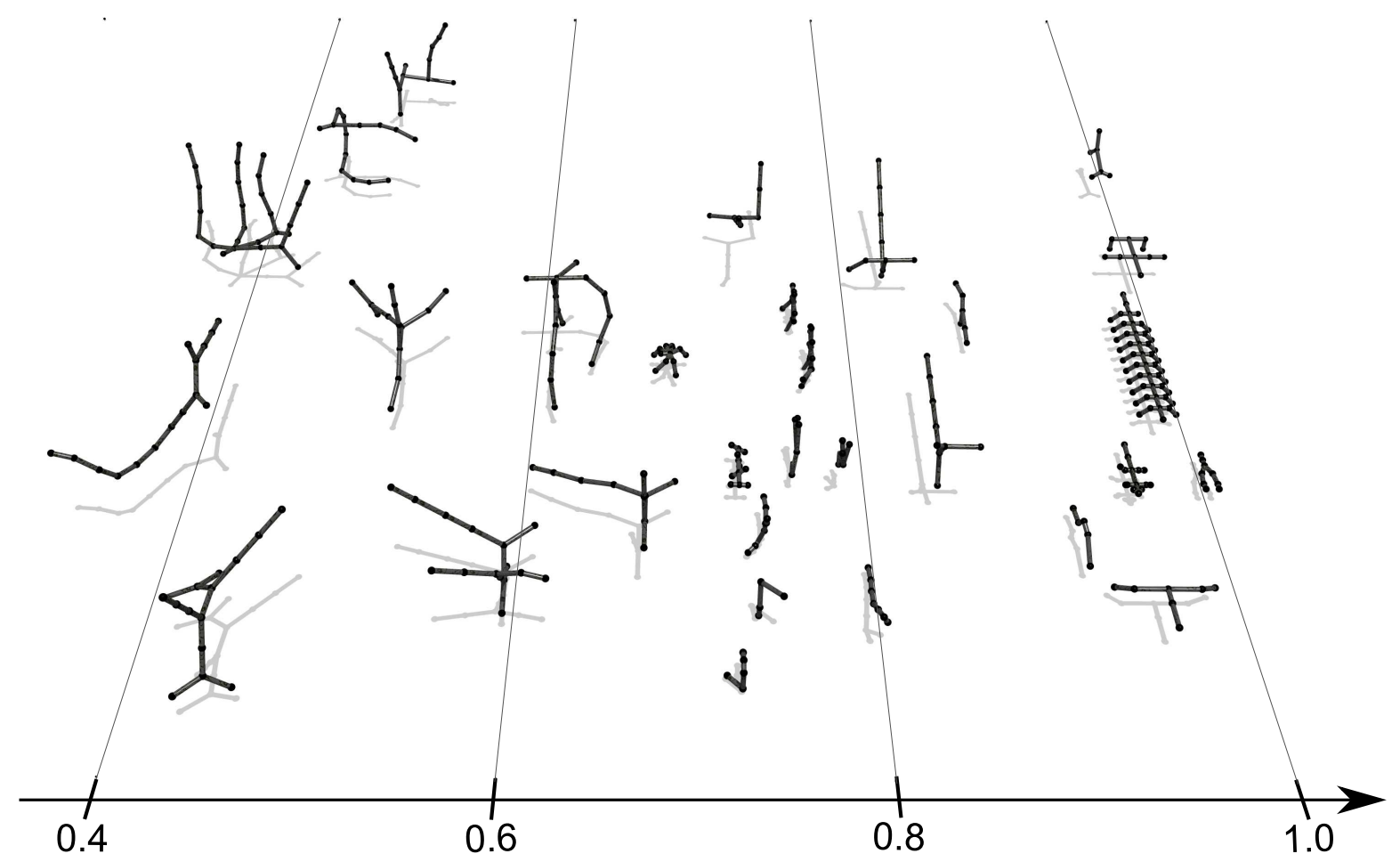

Figure 11: 30 diverse creatures arranged horizontally according to their values of symmetry (the most symmetrical on the right).

and the remaining 46 creatures were created in the process of evolution with no human assistance. Evolved constructs originated from various evolutionary processes oriented towards creature speed and creature height. Designed constructs served various purposes, most often efficient locomotion, specific mechanical properties or aesthetic shape.

Fig. 12 shows the distribution of symmetry values in the set of considered creatures. The vast majority $(92 \%)$ of designed creatures appeared to be symmetrical or nearly symmetrical (symmetry higher than 0.9 ). Moreover, $82 \%$ of designed creatures are completely symmetrical - with the symmetry value of 1.0 (see Fig. 13). Clearly, human designers prefer symmetry, and there were no human designs in the set with symmetry less than 0.6.

Although half of the evolved creatures also appeared highly symmetrical, symmetry of the rest is distributed fairly uniformly. It has to be noted that among evolved creatures with complete symmetry, many structures were very simple and, obviously, it is always easier to find a symmetry plane for such small creatures. Human designs with complete symmetry were more complex. Evolved creatures are shown in Fig. 14. The most asymmetric (albeit regular) creature is a spring-like individual — its value of symmetry is approximately 0.1 .

\section{Summary}

In this work, the numerical, continuous measure of symmetry for 3D stick creatures has been introduced. It was described in a formal mathematical way, and efficient algorithms were designed and implemented. The measure was then successfully applied to evolved and designed creatures taken from the Framsticks genotypes database. Experiments proved that the algorithms proposed here could be used to sort creatures according to their symmetry. A mixed set of individuals originating from human design and evolution revealed that symmetry dominates among human designs, and it is common in evolved constructs. 


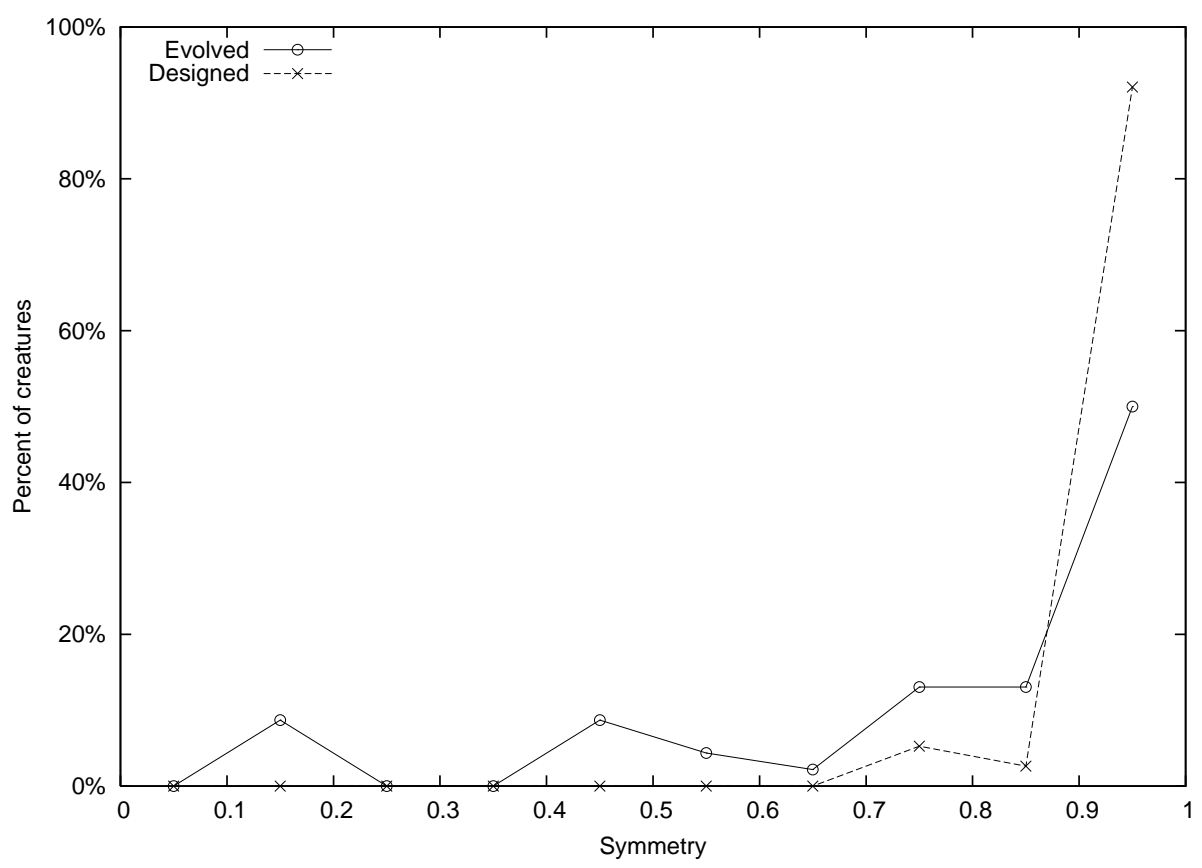

Figure 12: Distribution of symmetry values among 84 creatures (38 designed, 46 evolved). Each point in the graph denotes percentage of creatures having symmetry within a certain range (e.g. $(0.9,1])$. Points are connected for clarity.

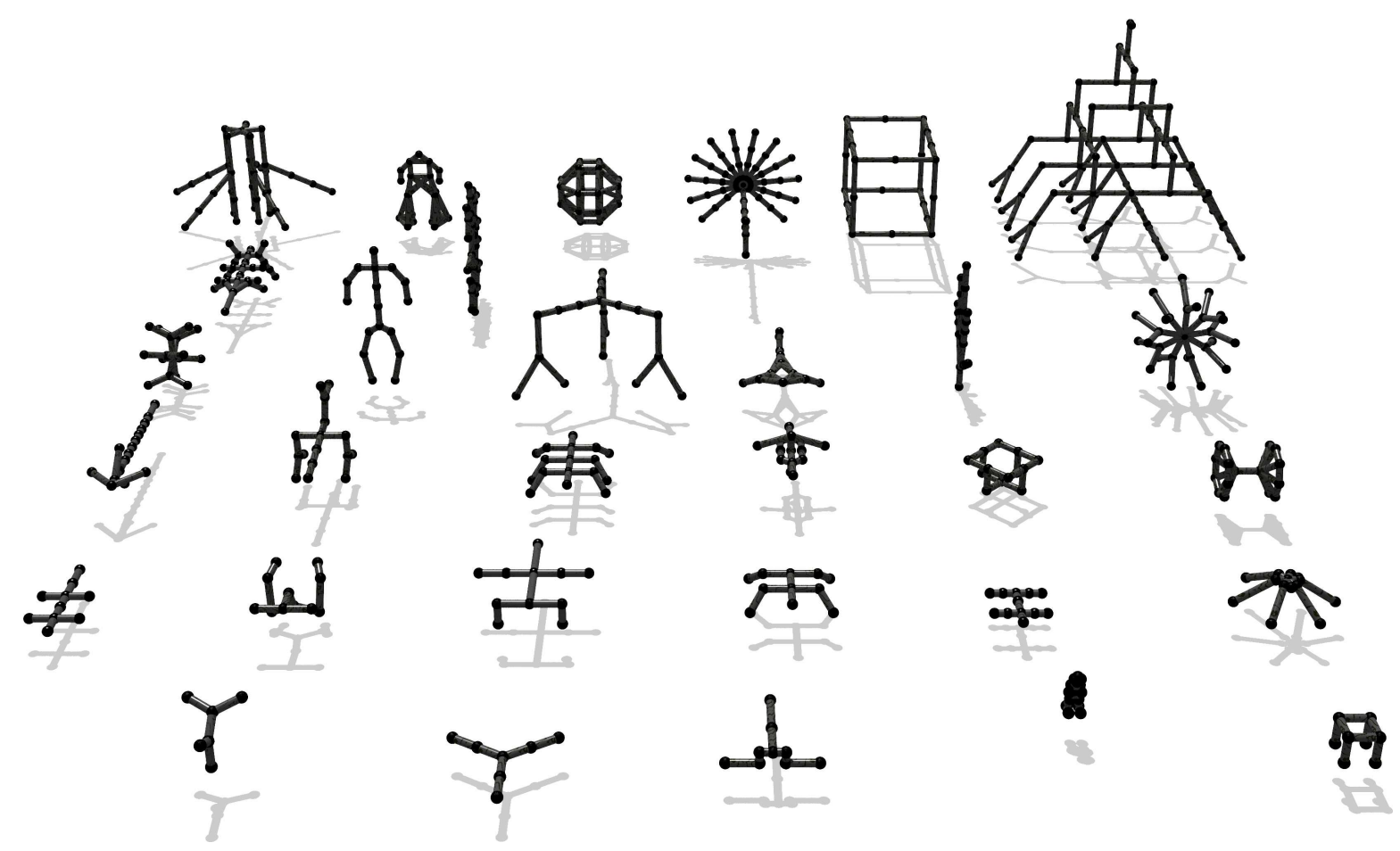

Figure 13: Designed creatures with symmetry of 1.0. 


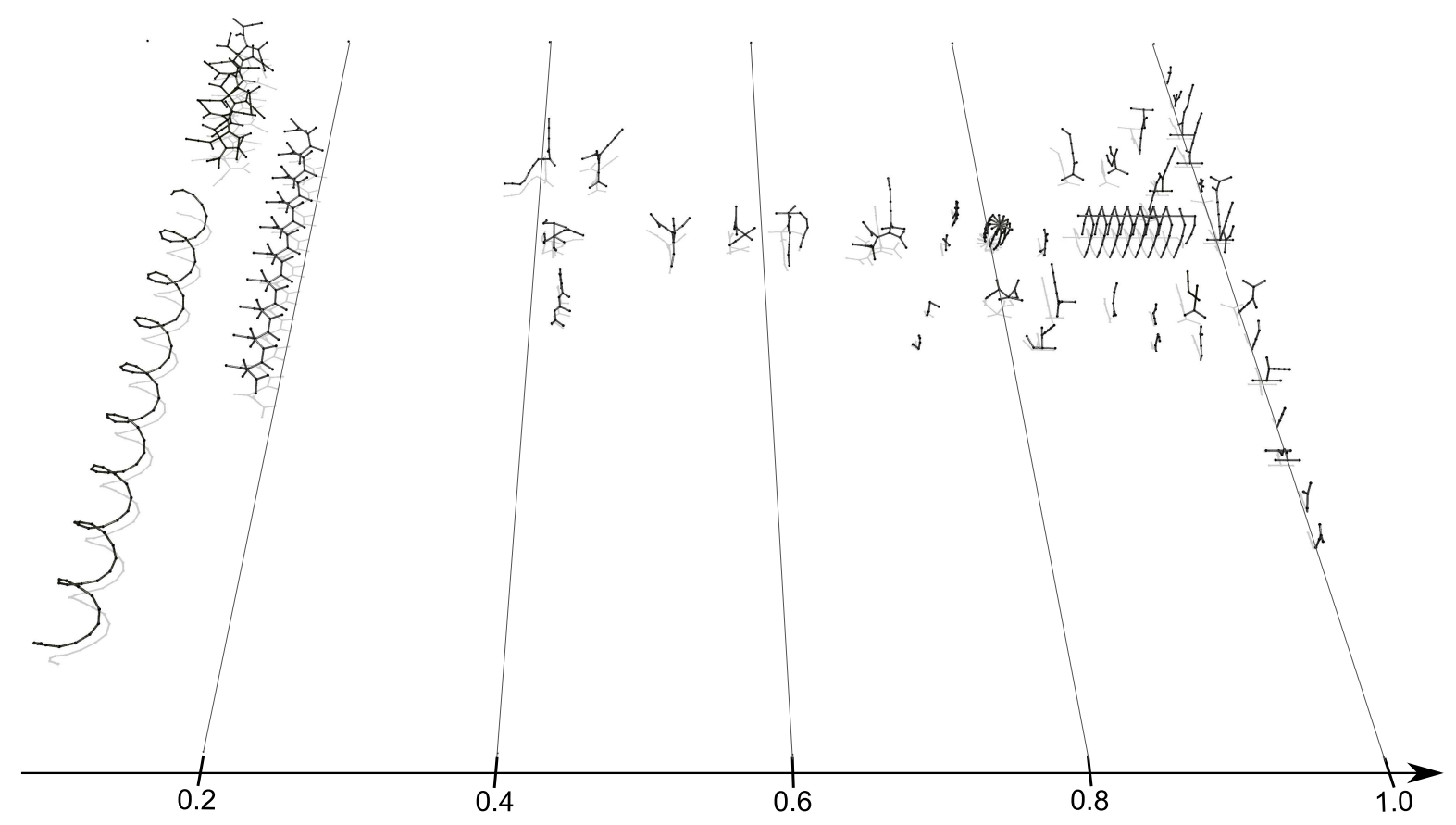

Figure 14: Evolved creatures. Constructs with the highest symmetry are usually simple.

Although it is difficult to objectively evaluate the quality of the measure, based on the experiments it is clear that more symmetrical creatures get higher symmetry values (The Common Sense Condition). The proposed symmetry measure also fulfills the three other important conditions stated in Sec. 3:

- The Symmetry Condition is fulfilled by the definition of the proposed measure.

- The Asymmetry Condition can not be verified objectively, but for the definitely asymmetrical spring-like creature (Fig. 14) our symmetry measure gives a value close to zero.

- The Scalability Condition is fulfilled by introduction of the proper similarity definition (Eq. 8).

It is hard to verify if The Proportional Difference Condition is fulfilled. It would be interesting to examine a correlation between a human sense of symmetry and the results of the measure proposed in this report. Then, it would be also possible to statistically verify The Common Sense Condition.

The proposed method is quite robust against the influence of the number of sticks (i.e. changing the number of sticks without any change in shape of a creature). Additional experiments that were performed suggest that symmetry values do not change significantly while increasing the number of sticks. Let us consider the creature shown in Fig. 1. Originally, its symmetry is 0.75 . When each stick is split into $n=2$ sticks (thus the total number of sticks doubles), the symmetry value does not change (see Fig. 15). For $n=3,4,5$, the value of symmetry decreases to $0.746,0.730$ and 0.713 , respectively. For higher values of $n$, the symmetry slowly increases. Therefore, even such extreme changes in the number of sticks influence only slightly the symmetry value.

Further research concerning the impact of symmetry on fitness of artificial creatures can help explain the process of biological evolution. For instance, it would be interesting to investigate for which objectives (speed and locomotion, predation, height, etc.) evolution promotes symmetrical creatures. An interesting question is whether symmetry is beneficial for creatures evolved spontaneously (i.e. with endogenous reproduction and no external fitness function).

The majority of biological living creatures are symmetrical, but it is not entirely clear how the property of symmetry is encoded in genotypes. For artificial creatures, diverse genetic encodings may exist (there are several encodings in the Framsticks system [5]). Some of them allow to evolve creatures 


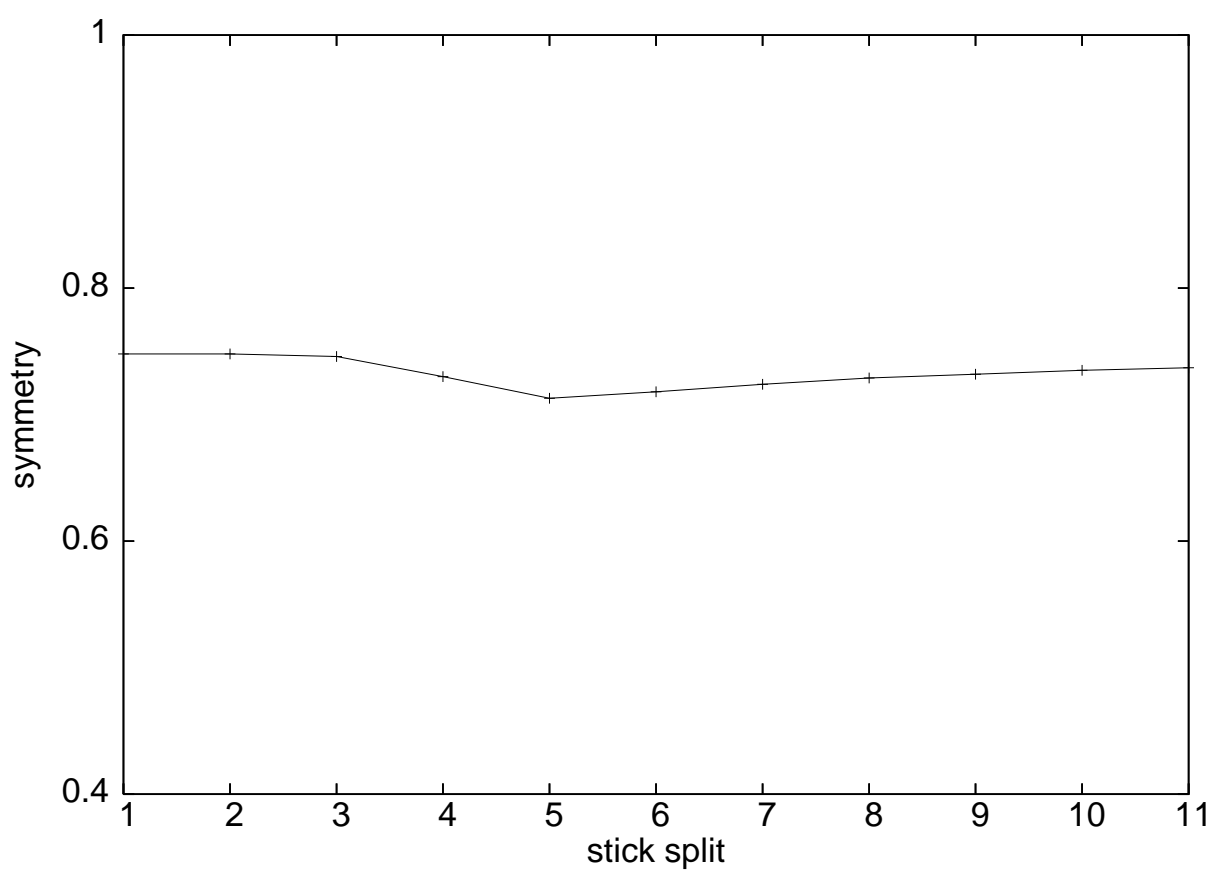

Figure 15: Minor influence of increased number of sticks on the symmetry value.

that are better adapted to certain tasks than others. We would like to investigate which encodings promote symmetry of evolved creatures and if it correlates with creature fitness. Symmetry can also be introduced as a component of the fitness formula to see if it helps in accomplishing evolutionary goals. Finally, a new encoding that preserves symmetry of creatures can be designed and compared with the existing ones.

In the experiments presented here, the implementation of the symmetry measure was used off-line (i.e. for static creatures), so the quality of results was most important, not the execution time. These high quality settings result in a long computation time for highly complex creatures (i.e. made of 100 sticks or more). For on-line experiments, when every evolved creature is tested for symmetry, a faster algorithm for finding best plane of symmetry would be better. The primary idea is to improve the algorithm that finds the best plane by introducing a better space search strategy (simulated annealing, for instance) instead of sampling the 3D grid as it is in the current implementation.

In this report we evaluated only static phenotypes (bodies just as they are defined by genotypes). The symmetry measure can be employed during the lifetime of a creature to estimate symmetry while an individual is in motion. This would allow investigations on how the plane of symmetry and the symmetry itself changes when a creature moves and bends some parts of its body. However, this is not trivial to study, because the plane of the highest symmetry can vary highly while the creature moves, as shown by the case of "Scorpion" and "Scorpion moving" in Fig. 10.

The plane of symmetry for biological organisms is in vast majority of cases consistent with their direction of movement. This phenomenon may also take place in artificial worlds and it is worth exploring, as some of the evolved animats surprise observers with their unusual methods of locomotion.

\section{Acknowledgements}

This work has been supported by the Ministry of Science and Higher Education, grant no. N N519 350533. 


\section{References}

[1] Balmford, A., Jones, I., \& Thomas, A. (1993). On avian asymmetry: evidence of natural selection for symmetrical tails and wings in birds. Procs. of the Royal Society of London B, 252, 245-251.

[2] G. Bock \& J. March (Eds.). (1991). Biological asymmetry and handednees. Willey and Sons, New York, NY.

[3] Bongard, J., \& Paul, C. (2000). Investigating morphological symmetry and locomotive efficiency using virtual embodied evolution. In J.-A. M. et al. (Ed.), From Animals to Animats: The Sixth International Conference on the Simulation of Adaptive Behaviour.

[4] Evans, M., Martins, T., \& Haley, M. (1994). The asymmetrical cost of tail elongation in redbilled streamertails. Procs. of the Royal Society of London B, 256, 97-103.

[5] Komosinski, M. (2005). Framsticks: A platform for modeling, simulating and evolving 3D creatures. In A. Adamatzky \& M. Komosinski (Eds.), Artificial Life Models in Software, chapter 2, (pp. 37-66). New York: Springer-Verlag.

[6] Komosinski, M., Koczyk, G., \& Kubiak, M. (2001). On estimating similarity of artificial and real organisms. Theory in Biosciences, 120(3-4), 271-286.

[7] Livio, M. (2005). The equation that couldn't be solved. how mathematical genius discovered the language of symmetry. Simon \& Schuster.

[8] Moller, A. (1990). Fluctuating asymmetry in male sexual ornaments may reliably reveal male quality. Animal Behaviour, 40, 1185-1187.

[9] Moller, A. (1992). Female swallow preference for symmetrical male sexual ornaments. Nature, $357,238-240$.

[10] Norberg, R. (1977). Occurrence and independent evolution of bilateral ear asymmetry in owls and implications on owl taxonomy. Phil. Trans. of Royal Soc. of London B, 280, 375-408.

[11] Rhodes, G., Proffitt, F., Grady, J., \& Sumich, A. (1998). Facial symmetry and the perception of beauty. Psychonomic Bulletin and Review, 5, 659-669.

[12] Thomas, A. (1993). The aerodynamic cost of asymmetry in the wings and tail of birds: asymmetric birds can't fly round tight corners. Procs. of the Royal Society of London B, 254, 181-189.

[13] Zabrodsky, H., Peleg, S., \& Avnir, D. (1992). Continuous symmetry measures. J. Am. Chem. Soc., 114, 7843-7851.

[14] Zabrodsky, H., Peleg, S., \& Avnir, D. (1993). Continuous symmetry measures. 2. symmetry groups and the tetrahedron. J. Am. Chem. Soc., 115, 8278-8289.

[15] Zabrodsky, H., Peleg, S., \& Avnir, D. (1995). Symmetry as a continuous feature. IEEE Trans. on PAMI, 17, 1154-1166.

[16] Zaidel, D., Aarde, S., \& Baig, K. (2005). Appearance of symmetry, beauty, and health in human faces. Brain and Cognition, 57(3), 261-263. 